\title{
Electrolysis for hydrogen production
}

\author{
By Prachi Patel \\ Feature Editor: Kathy Ayers
}

$T^{2}$ he lightest element has carried a heavy burden for half a century. Expectations for the hydrogen economy, first proposed in the 1970s, have been high. But hydrogen as a renewable, low-carbon fuel for vehicles, heating, and energy storage has remained evasive, held back by high costs, low efficiency, and a lack of infrastructure and storage technologies.

Yet change is in the air. The International Energy Agency (IEA) calls this a year of "unprecedented momentum" for hydrogen in its June 2019 report, The Future of Hydrogen. Past initiatives focused on hydrogen fuel cells for vehicles. But this time, things are different, the report noted.

Pressure to decarbonize the global economy has nudged policymakers and companies to look at hydrogen as a way to tackle emissions across a range of sectors beyond transportation. The cost to make and use hydrogen is falling. Renewables have become a key driver and beneficiary: as their share for power generation goes up, hydrogen could be the lowest-cost option for storing gigawatts of surplus solar and wind power for a long time, even compared to batteries. "It's no longer a question of whether hydrogen will be part of an energy system, it's what fraction it will comprise," said Brian Pivovar, Fuel Cell Group Manager, Chemistry and Nanoscience Center, National Renewable Energy Laboratory (NREL).

However, nearly all of the 70 million metric tons of hydrogen used every year - mainly for ammonia production and petroleum refining - is currently made by cracking natural gas via steam reforming, which is an energy-intensive, carbon-spewing method.

For hydrogen to fulfill its promise as a clean fuel, there is a need to switch its production from gray to green. Age-old electrolysis, which splits water into pure hydrogen and oxygen by passing electricity through it, is one of the most promising technologies to enable that. Provided, of course, that electricity used for the systems comes from renewables. "If you have a surplus of electricity coming from renewables in the future, it makes sense to push those inexpensive electrons into an electrolyzer to make hydrogen," said Marcelo Carmo, head of electrochemistry at Forschungszentrum Jülich $\mathrm{GmbH}$ in Germany.

The problem is that hydrogen from electrolysis today is twice as expensive as that from steam reforming, which produces hydrogen for USD $\$ 2 / \mathrm{kg}$. Electricity makes up $80 \%$ of the cost of electrolic hydrogen. But electrolyzer costs will come to the forefront as renewables provide cheap electricity in the future. Materials scientists and engineers are now working on better catalysts, membranes, and structural components, as well as manufacturing methods, that could chip away at capital and operating costs and help make hydrogen from water splitting a part of tomorrow's low-carbon picture.

Nine countries now have national roadmaps for hydrogen. Some experimental projects in Europe are looking into delivering hydrogen for heating buildings through the existing natural-gas infrastructure. About 12,000 fuel-cell cars are now on the road. Many companies are rolling out hydrogen-powered trucks and forklifts, and Arizona startup Nikola Motors plans to build a crosscountry network of hydrogen fueling stations.

Electrolysis accounts for only $4 \%$ of hydrogen produced today, but the technology has seen a surge in interest in recent years. The number and size of installations are increasing. More than $20 \mathrm{MW}$ of electrolyzer capacity came online in 2018 . The typical installation size has gone up from $0.1 \mathrm{MW}$ to $1 \mathrm{MW}$ since 2009 . A 10-MW electrolyzer in Germany and a 20-MW electrolyzer in Canada are now under construction.

Two electrolyzer technologies are commercially available on a large scale: proton-exchange membrane (PEM) and alkaline electrolysis. Both work at low temperatures of $50-80^{\circ} \mathrm{C}$. Hightemperature electrolysis, which is based on solid-oxide separators, faces cost and durability challenges, but a few companies have plans to improve and commercialize the technology. Much of the ongoing research in electrolysis aims to reduce cost and increase efficiency and durability.

Alkaline electrolyzers, used since the 1920s, are the cheapest and most proven water-splitting technology. They have efficiencies of about $63-70 \%$ and can run for $60,000-90,000 \mathrm{~h}$. These systems typically use nickel electrodes and catalysts and a porous polymer membrane loaded with a liquid sodium or potassium hydroxide electrolyte, which transports hydroxyl ions from the cathode to the anode. "I think the potential for low-cost and fully scalable technology is very high with alkaline systems," said Jens Oluf Jensen, a professor at the Technical University of Denmark.

Membranes today are relatively thick at a few millimeters, to better separate the oxygen and hydrogen. The high electrical resistance of these thick membranes can bring down efficiency. Jensen and his colleagues are making thinner nonporous polymer membranes that separate just as well. "It works very well but is not long-term stable, so we are trying to improve stability," he said.

The market for PEM systems is growing fast, with many commercial electrolyzer units being constructed or planned. First made by General Electric in the 1950s for the space program, these electrolyzers can have about half the footprint of alkaline systems, owing to their thin polymer electrolyte. However, their use of platinum- and iridium-based catalysts at the electrodes to handle the harsh acidic conditions and that of titanium as a structural material makes them more expensive compared to alkaline electrolyzers. 
At NREL, Pivovar and his colleagues are trying to improve the performance, cost, and durability of PEM electrolyzers by testing novel materials and developing new components, and understanding factors that affect performance and degradation.

One big push is to reduce the catalyst at the electrodes. Efforts include using high-surface-area catalysts or oxides of multiple metals. Today's PEM systems use several milligrams of noble metal catalysts per square centimeter. The extra material helps combat the losses because of high voltages, harsh chemical conditions, and mechanical stresses. Using iridium nanoparticles on tungsten-doped titanium oxide, the NREL team was able to reduce iridium loading from $70 \%$ to $25 \%$. Lowering catalyst loading does not accelerate material loss mechanisms, such as iridium dissolution or electrode thinning, said NREL's Shaun Alia, "but it does dramatically accelerate how quickly those losses are observed."

Others are also experimenting with iridium nanoparticles, nanowires, and nanotubes. "It's a lot of materials science," said Carmo. "How the electrodes are fabricated and how the materials are nanostructured. It's about maximizing surface area, electron and ion conductivity, and water transport; all these things are happening inside electrodes."

"The next step will be to get rid of iridium," he said. Iridium is the least abundant material in the earth's crust, and eliminating it would be critical if these electrolyzers are going to expand to gigawatt scales.

Meanwhile, researchers are trying to replace titanium structure materials, which make up a quarter of the cost of an electrolyzer stack, with titanium-coated steel or carbon composites.

A key challenge for PEM systems is also to reduce membrane thickness, while preventing hydrogen and oxygen to pass through. Commercial membranes are made of perfluoroalkyl sulfonic acid, such as Nafion. But researchers are looking at alternative materials, such as hydrocarbon polyarylene-based and fluoropolymer-based membranes, which are less permeable to gas. These could be less expensive, but researchers need to prove their chemical and mechanical stability in electrolyzers. "The challenge has been that any benefit to performance and cost either doesn't translate to the device or comes at the expense of durability," said Alia.

One exciting approach is to combine the best of alkaline and PEM systems. The idea is to use a thin, highly conductive solid polymer membrane in alkaline systems, where they can conduct hydroxyl ions instead of protons. It is more difficult than it sounds. Traditional ion-exchange membranes have immobilized ions on small side chains, which help to conduct ions. "That works very well in acidic systems, but it is very difficult to develop materials that conduct well and are stable in alkaline electrolytes," said Jensen. The electrolytes end up harming the quaternary ammonium ions on the side chains.

Jensen, Carmo, and others reported a breakthrough: an ionsolvating sodium hydroxide-doped polybenzimidazole membrane that has a more homogenous chemical structure. Prototype cells with this membrane and nickel-based electrodes show current densities matching state-of-the-art PEM electrolyzers without the use of platinum group catalysts, although they are less stable and efficient.

High-temperature solid-oxide electrolysis cells (SOECs) also ditch expensive catalysts. These solid-state devices usually have nickel electrodes and a zirconium oxide ceramic electrolyte that conducts ions. They use steam instead of water for electrolysis and boast high electrical efficiency. "Thermodynamically, it's much easier to split steam, so the requirement for electrical energy is lower at high temperatures," said Olga Marina, chief scientist in the Energy Processes \& Materials Division at Pacific Northwest National Laboratory (PNNL). "Also reaction kinetics are much faster at high temperatures, so we can produce hydrogen faster."

Plus, SOEC electrolyzers can also take carbon dioxide in addition with hydrogen to produce synthetic gas, a useful precursor for liquid fuels. But the blistering temperatures of $700-800^{\circ} \mathrm{C}$ wear down metal parts. And generating heat uses a lot of energy. Tests of prototype systems at PNNL show that the technology is feasible and reliable, said Marina. "We can demonstrate great performance, but haven't demonstrated durability and stability at that performance."

Along with materials improvements, economies of scale should reduce electrolyzer costs. A gradual shift to larger systems that combine multiple electrolyzer stacks is expected to reduce the cost of alkaline systems by $20 \%$ and of PEM systems by $40 \%$, according to the IEA report. Marina said small-volume manufacturing is the biggest culprit behind the high cost of SOECs. "Additive manufacturing should help produce system components at a higher volume. Robotics and new tools should cut labor cost and bring down the price of systems. And then we can prove durability."

The trajectory of fuel cells offers hope for electrolyzers. Thirty years ago, fuel cells had similarly thick Nafion membranes and electrodes with high catalyst loading. Today, fuel-cell vehicles are on the roads. They use 10-15- $\mu \mathrm{m}$-thick mechanically reinforced and chemically stabilized membranes, and alloy catalysts with low loadings of $0.25 \mathrm{mg} / \mathrm{cm}^{2}$. Fuel-cell vehicles in 2002 were projected to cost USD $\$ 300 / \mathrm{kW}$ and today are projected to be USD\$45, said Pivovar. "That's because of billions of dollars of investment in materials-related research." 